deux catégories d'équilibre. La comparaison des coefficients de régression des divers départements et des taux de consanguinité de ces départements montre que ces deux caractéristiques sont étroitement liées, avec un coefficient de corrélation de rang très significatif et négatif, égal à $-0,76$. Tout semblerait se passer comme si aux effets élémentaires des divers gènes venait s'ajouter un effet d'inter-action de sens opposé; autrement dit, l'effet défavorable global de plusieurs gènes serait inférieur à la somme des effets défavorables de chacun d'eux. On peut penser qu'une part de la très forte corrélation négative correspond à un autre phénomène biologique : plus une population est endogame, plus les gènes défavorables recessifs peuvent s'exprimer et être soumis à une élimination par sélection. A la longue, les populations les plus endogames sont donc celles qui ont le plus "épuré "leur patrimoine génétique et sont devenues les moins sensibles aux effets de la consanguinité.

\title{
CALCUL DES PARAMìtTRES GÉNÉTIQUES ET ÉTABLISSEMENT D'INDEX DE SÉLECTION SUR PLUSIEURS CARACTÈRES
}

\author{
B. Poujardieu, R. Rouvier, Michelle Briend, J.-M. I.eclaire. - Station de Génétique \\ quantitative et appliquée, C.N.R.Z., 78-Jouy-en-Josas
}

La sélection des animaux domestiques s'opère souvent sur des échantillons composés de familles et de sous-familles, suivant une classification hiérarchisée. L'objet de la sélection est d'abord d'estimer la valeur génétique additive des animaux susceptibles d'être gardés comme reproducteurs. Cette estimation s'effectue classiquement par une fonction linéaire des valeurs phénotypiques individuelles, moyennes de sous-famille et de famille, pour les caractères concernés. Les conditions posées pour cette estimation peuvent avoir trait à la variabilité ou à l'intérêt économique des caractères. Il convient de calculer en premier lieu la matrice des variances et covariances génotypiques et phénotypiques des caractères. Nous avons écrit en Fortran IV, en vue d'une utilisation sur ordinateur IBM 360-50, une séquence de programmes permettant de résoudre ces diverses opérations dans les cas suivants de calcul des matrices des composantes des variances et covariances, des corrélations entre classes, des pourcentages de variance dus à chaque facteur, pour 6 facteurs hiérarchisés et 30 variables au maximum, et des nombres inégaux de données par cellule. Ce programme utilise 50000 positions de mémoire en unité centrale. Les résultats peuvent être directement repris par un programme de calcul d'index de sélection sur plusieurs caractères. L'index de sélection est un estimateur d'un génotype global défini comme une combinaison linéaire des valeurs génétiques additives des caractères considérés de coefficients donnés. Les situations suivantes sont considérées dans le programme de calcul d'index : sélection massale; sélection combinée sur la valeur individuelle et les moyennes des familles de demi-frères ou de pleins frères, ou bien de demi-frères et de pleins frères (les valeurs individuelles des animaux pour lesquels on calcule l'index sont inclues dans ces moyennes de familles); sélection sur descendance ou sur collatéraux pour des familles de demi-frères ou de pleins frères, sélection sur collatéraux répartis en familles de demi-frères et de pleins frères. Les formules d'index utilisées supposent que les pères, les mères et les enfants ne sont pas consanguins. Les effectifs de familles et sous-familles sont supposés constants et peuvent être fixés par carte contrôle. Ce programme, qui utilise 57000 positions de mémoire en unité centrale, calcule dans tous les cas les coefficients de l'équation d'index, la variance de l'index, les espérances des progrès génétiques de chacun des caractères et du génotype global. Il peut en outre calculer les valeurs individuelles des index. Pour améliorer la précision des valeurs calculées, nous nous sommes ramenés, par partition, à des inversions de matrices dont l'ordre est au plus égal au nombre de variables considérées dans l'index. La méthode biométrique utilisée pour la décomposition des variances et covariances est décrite par KEMPTHORNE (1957). Les modèles d'estimation de la valeur génétique additive ont été étudiés par ROUVIER (1969). 